\title{
miR-193a Directly Targets PSEN1 and Inhibits Gastric Cancer Cell Growth, the Activation of PI3K/Akt Signaling Pathway, and the Epithelial-to-Mesenchymal Transition
}

\author{
Xuemei Pan, ${ }^{1}$ Ting Zhao, ${ }^{1}$ Saisai Mu, ${ }^{2}$ and Shouchuan Li ${ }^{2}{ }^{2}$ \\ ${ }^{1}$ General Surgery Ward I, Qingdao Hospital of Traditional Chinese Medicine, Qingdao Hiser Hospital, Qingdao 266000, China \\ ${ }^{2}$ General Surgery Ward III, Qingdao Hospital of Traditional Chinese Medicine, Qingdao Hiser Hospital, Qingdao 266000, China \\ Correspondence should be addressed to Shouchuan Li; lishouchuan@qdzyhospital.cn
}

Received 31 May 2021; Accepted 5 July 2021; Published 15 July 2021

Academic Editor: Muhammad Wasim Khan

Copyright (c) 2021 Xuemei Pan et al. This is an open access article distributed under the Creative Commons Attribution License, which permits unrestricted use, distribution, and reproduction in any medium, provided the original work is properly cited.

Background. Gastric cancer, a kind of gastrointestinal malignancy, is the second type of leading death cancer. miR-193a is a key tumor suppressor in several diseases. PSEN1 is mainly related to Alzheimer's disease and may be involved in the cleavage of the Notch receptor. Material and Methods. RT-PCR and western blot were applied to evaluate miR-193a and the expression level of PSEN1. Luciferase reporter assay was applied to verify whether PSEN1 was a target of miR-193a. The Kaplan-Meier method was employed to calculate the 5-year overall survival of gastric cancer patients. Results. miR-193a was downregulated in gastric cancer tissues and cell lines, and downregulation of miR-193a predicted poor 5-year overall survival of gastric cancer. miR-193a inhibited the proliferation and the activation of the PI3K/AKT signaling pathway in gastric cancer cells. miR-193a inhibited gastric cancer tumor growth in vivo. miR-193a impaired cell invasion and epithelial-to-mesenchymal transition (EMT) in HGC-27 cells. In addition, PSEN1 was a direct target of miR-193a and PSEN1 reversed partial functions of miR-193a in cell proliferation and invasion. Conclusion. miR-193a prominently decreased the proliferation, invasion, and activation of the PI3K/Akt signaling pathway and the abilities of epithelial-to-mesenchymal transition in gastric cancer cells. The newly identified miR-193a/PSEN1 axis provides novel insight into the pathogenesis of gastric cancer.

\section{Introduction}

Gastric cancer (GC), the second leading death cancer, is a kind of gastrointestinal malignancy [1]. Lamentably, gastric cancers patients at an early stage are difficult to diagnose due to no specific symptoms [2]. Thus, exploring new biomarkers for the early diagnosis and treatment of gastric cancer is urgent.

Micro-RNAs (miRNAs), short noncoding RNAs, could cause mRNA degradation or translational inhibition through binding to target mRNAs $3^{\prime}$-UTR at the posttranscriptional level $[3,4]$. Recently, accumulating evidence indicated that miRNAs play a vital role in tumor progression in gastric cancer, including proliferation, metastasis, and apoptosis [5-7]. miR-193a acted as a critical tumor suppressor in various cancers, including colon cancer, acute myeloid leukemia, bladder cancer, and malignant pleural mesothelioma [8-11]. In non-small cell lung cancer, Yu et al. illuminated that miR-193a suppressed cell migration, invasion, and EMT [12]. Similarly, Fang et al. demonstrated that miR-193a inhibited cell proliferation and metastasis in pancreatic cancer [13]. Therefore, we strongly believe miR193a inhibited cell proliferation and invasion in gastric cancer.

Presenilin-1 (PSEN1), a primary component of the $\gamma$-secretase complex, is mainly related to Alzheimer's disease and may be involved in the cleavage of the Notch receptor $[14,15]$. The mutation of PSEN1 was associated with spasticity and parkinsonism in the dominant Alzheimer's family [16]. Zhao et al. indicated that PSEN1 overexpression results in attenuated phagocytic uptake of $A \beta$ by microglia and regulated intracellular trafficking and 
pathophysiological function in myeloid cells [17]. In addition, Meng et al. illuminated that PSEN1 was a target gene of miR-193a and miR-193a regulated chemoradiation resistance through PSEN1 in oesophageal cancer [18].

In this present study, we aimed to investigate the biological effect of miR-193a on gastric cancer. To translate these findings to clinical practice, it is essential to explore the molecular mechanism of miR-193a in the development of gastric cancer.

\section{Materials and Methods}

2.1. Patients and Tissue Samples. We selected 50 patients that underwent an operation at Qingdao Hospital of Traditional Chinese Medicine, Qingdao, China, and obtained 50 pairs of gastric cancer tissues and corresponding peritumoral normal tissues. The specimens were immediately frozen in liquid nitrogen and followed storage at $-80^{\circ} \mathrm{C}$. Informed consent was obtained from each patient and this investigation was approved by the ethics committee of the Qingdao Hospital of Traditional Chinese Medicine, Qingdao, China.

2.2. Cell Lines and Culture Conditions. We obtained two human gastric cancer cells HGC-27 and MGC-803 and a normal gastric epithelial cell GES-1 from American Type Culture Collection (ATCC, Rockville, MD, USA). RPMI1640 (Gibco-BRL; Rockville, MD, USA) medium contained with $10 \%$ FBS (Haoyang Biological, Tianjin, China) were conducted to culture the cells at $37^{\circ} \mathrm{C}$ with $5 \% \mathrm{CO}_{2}$.

2.3. Plasmid Construction and Transfection. We purchased the miR-193a mimic and miR-193a inhibitor oligo fragments from Gene-Pharma (Shanghai, China), which was conducted to upregulate or downregulate miR-193a. HGC27 cells were seeded in a 6-well plate, and the transfection was performed using Lipofectamine 2000 (Invitrogen, Carlsbad, USA) reagent as described previously [19]. In brief, the Opti-MEM/reduced serum medium (Thermo Fisher Scientific, Shanghai, China) was employed to dilute the Lipofectamine 2000 reagent and the oligo fragments, respectively. After mixing the two solutions, the mixture was added to the cells. For the transiently transfected cells, the cells were harvest after $48 \mathrm{~h}$. On the contrary, for the cells with stable transfection, we applied Geneticin (G418, Thermo Scientific, Shanghai, China) to select.

2.4. RNA Extraction and Quantitative Real-Time PCR. TRIzol reagent (Invitrogen) and mirVana miRNA Isolation Kit (Thermo, Shanghai, China) were employed to extract the total RNAs and total miRNAs, respectively. The first cDNA chains of mRNA and miRNA were synthesized by the Prime Script RT Reagent Kit (Takara Biotechnology Co., Ltd., Dalian, China) and the miRNA Reverse Transcription Kit (Life Technologies, Foster, CA, USA), respectively. The qPCR was performed by using the SYBR Premix Ex Taq (Takara) and MystiCq microRNA qPCR Assay Primer (Sigma, Missouri, USA) on Applied
Biosystems StepOnePlus ${ }^{\mathrm{TM}}$ Real-Time PCR System. The normalization for miR-193a and PIK3CG was used as U6 and GAPDH, respectively. The primers were miR-193a F: $5^{\prime}$-ACTGGCCTACAAAGTCCCAGT-3', R: $5^{\prime}$-GTGC AGGGTCCGAGGT-3'; U6 F: 5'-CTTCGGCAGCACATATAC-3', R: 5'-GAACGCTTCACGAATTTGC-3'; PSEN1 F: 5'-TATGGCAGAAGGAGACCCG-3', R: 5' CCATTCCTCACTGAACCCG-3'; GAPDH F: $5^{\prime}$ AAGGTGAAGGTCGGAGTCAA-3', R: $5^{\prime}$-AATGAAGGGGTCATTGATGG-3’.

2.5. Western Blot Analysis. RIPA Lysis Buffer (Sigma, USA) containing 10\% PMSF (Sigma, USA) was conducted to lyse the HGC-27 cells on ice for $30 \mathrm{~min}$. We centrifuged the protein solving liquid at $4^{\circ} \mathrm{C}$ for $20 \mathrm{~min}$ with $12,000 \times \mathrm{g}$ speeds and then collected the supernatants. We separated the proteins by electrophoresis utilized 10\% SDS-PAGE and then transferred the blots on a PVDF membrane (Millipore, USA). After blocking by $5 \%$ fat-free milk in TBST buffer at room temperature for $1 \mathrm{~h}$, the blots were incubated with the primary antibodies at $4^{\circ} \mathrm{C}$ overnight. The membrane was incubated by primary antibodies, including PSEN1 (1 :1000; Abcam, Cambridge, USA), EMT proteins (E-cadherin (1: 1000; Abcam), and N-cadherin (1:1000; Abcam) and the proteins on the PI3K pathway (p-PI3K $(1: 1000$, Cell Signaling, San Jose, CA, USA), PI3K (1:1000, Cell Signaling), p-AKT ( $1: 1000$, Cell Signaling), AKT $(1: 1000$, Cell Signaling)), and GAPDH (1:1000, Santa Cruz, Shanghai, China). Subsequently, we incubated the membranes by antirabbit or mouse HRP-conjugated secondary antibody for $2 \mathrm{~h}$ at room temperature. In the end, Enhanced Chemiluminescence (ECL, Pharmacia Biotech, Arlington, USA) was employed to evaluate the signals.

2.6. CCK-8 Assay. The cell proliferative ability was conducted by Cell Counting Kit-8 (CCK8, Dojindo, Japan) [19]. HGC-27 cells were seeded in a 96-well plate and cultured for $24 \mathrm{~h}, 48 \mathrm{~h}, 72 \mathrm{~h}$, or $96 \mathrm{~h}$ at $37^{\circ} \mathrm{C}$ incubator. After being cultured, $10 \mu \mathrm{l}$ of CCK8 solutions was added to each well and cultured at $37^{\circ} \mathrm{C}$ for $1 \mathrm{~h}$; then, the absorbance was assessed at $450 \mathrm{~nm}$ using a microplate reader (BioTek, Winooski, USA).

2.7. Transwell Assays. The cell invasive ability was measured by transwell insert ( $8 \mu \mathrm{m}$ membrane, Corning, Cambridge, MA) with matrigel (BD Biosciences) covered [19]. The inserts were put in a 24-well plate to form the upper and lower chambers. The $200 \mu \mathrm{l}$ HGC-27 cells' suspension was added in the upper chamber, which was suspended by RPMI-1640 medium without PBS. Meanwhile, the lower chamber was filled with a $500 \mu \mathrm{l}$ RPMI-1640 medium containing 15\% FBS. After incubating the cells at $37^{\circ} \mathrm{C}$ for $24 \mathrm{~h}$, the noninvasive cells on the upper surface were removed using a cotton swab. For the invaded cells, they were fixed and then stained using $4 \%$ paraformaldehyde and $10 \%$ crystal violet in sequence; and then, the cells were counted under the microscope (Olympus Corporation, Tokyo, Japan). 


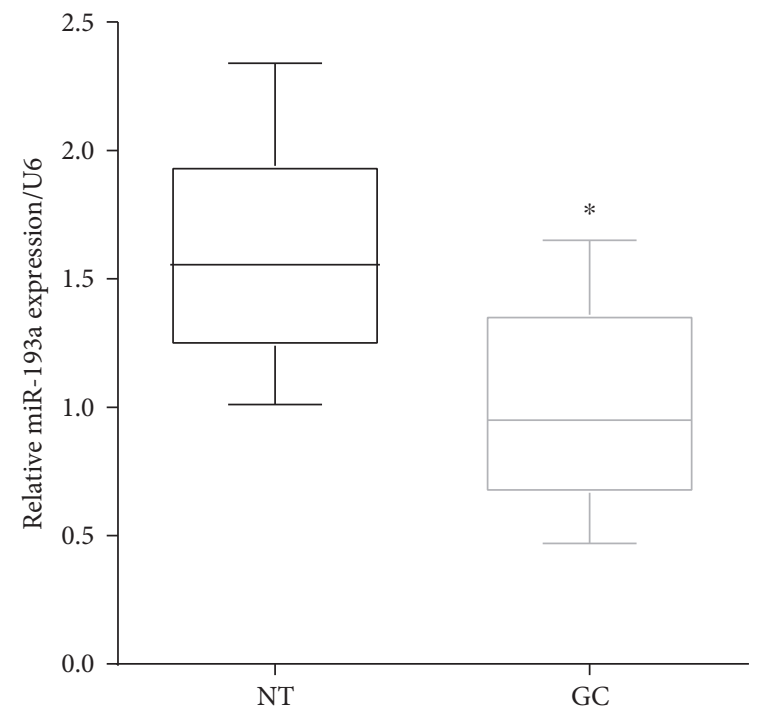

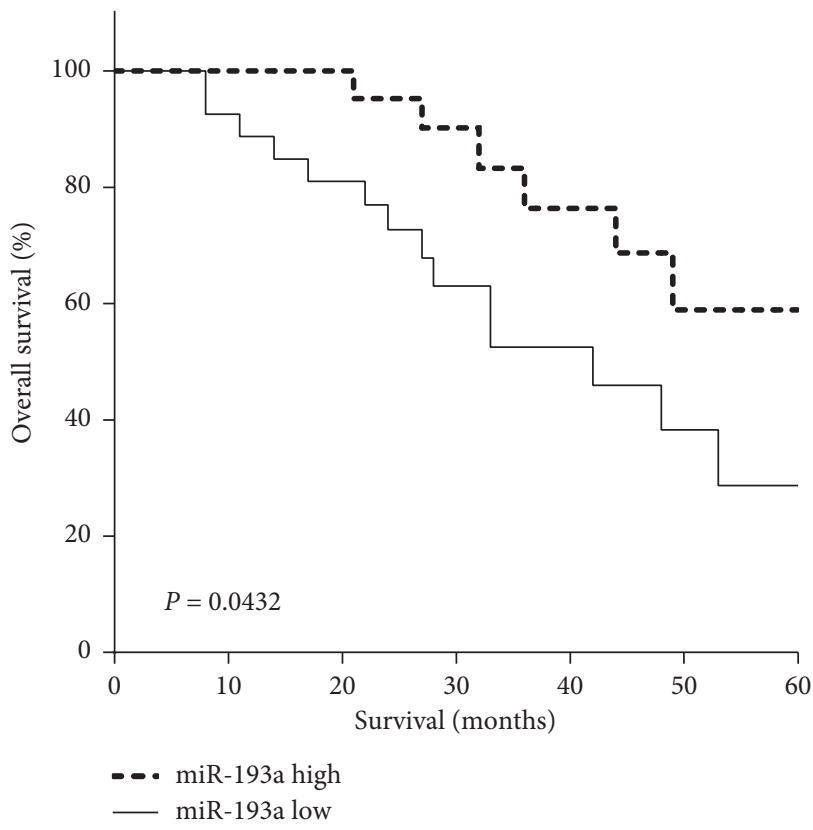

(b)

FIGURE 1: Downregulation of miR-193a predicts poor prognosis of gastric cancer. (a) miR-193a expression was low in GC tissues versus corresponding peritumoral normal tissues. (b) Low expression of miR-193a predicted poor prognosis of gastric cancer patients. ${ }^{*} P<0.05$.

2.8. Dual-Luciferase Reporter Assay. TargetScan predicted PSEN1 was a target gene of miR-193a, and the binding site was located at $697-703$ on PSEN1 mRNA 3'-UTR. To verify whether miR-193a directly binds to PIK3CG mRNA 3'UTR, the binding sequences were mutated from GGCCAGU to CCGGUCA and inserted in pmirGLO luciferase reporter vector. The HGC-27 cells were cotransfected with miR-193a mimic and the wild-type $3^{\prime}$-UTR or the mutant $3^{\prime}$-UTR of PSEN1. We utilized the dual-luciferase reporter assay system (Promega) to evaluate the firefly luciferase activity with Renilla luciferase activity as the normalization.

2.9. Tumor Xenograft Model in Nude Mice. The 4-week-old nude mice were purchased from Charles River Laboratories (Beijing, China). The nude mice were randomly classified into two groups, miR-193a mimic miR-193a control groups, with two mice in each group. HGC-27 cells were inoculated into the nude mice through subcutaneous injection. The mice were raised in the same environment and were observed every week. We measured and recorded the tumor lengths and widths and then calculated the tumor volume every three days. All animal experiments were performed in the animal laboratory center of Qingdao Hospital of Traditional Chinese Medicine and approved by the Care and Use Committee of Qingdao Hospital of Traditional Chinese Medicine.

2.10. Statistical Analysis. All the data were analyzed by SPSS statistical software version 16.0 (SPSS, Chicago, IL, USA), which were presented as the mean \pm standard deviation. The significance between two groups and multiple groups was compared by a two-tailed Student's $t$-test and one-way analysis of variance followed by an LSD test. $P$ value $<0.05$ was found to be statistically significant.

\section{Results}

3.1. Downregulation of miR-193a Predicts Poor Prognosis of Gastric Cancer. We evaluated the mRNA levels of miR-193a in 50 pairs of gastric cancer and peritumoral normal tissues. As expected, the expression of miR-193a was lower in GC tissues than that in the corresponding peritumoral normal tissues $(P<0.05)$ (Figure $1(\mathrm{a}))$. According to the median level of miR-193a, patients were divided into a low expression group and a high expression group [20]. Results showed that high expression of miR-193a was associated with poor prognosis in gastric cancer $(P<0.05)$ (Figure 1(b)).

3.2. miR-193a Suppresses Cell Proliferation and Inhibits the Activation of PI3K/Akt Signaling Pathway in Gastric Cancer Cells. We calculated miR-193a expression in two gastric cancer cells (HGC-27 and MGC-803) and a normal epithelial cell GES-1. The same as the tissues, miR-193a was low expressed in GES-1 cells versus HGC-27 $(P<0.01)$ and MGC-803 $(P<0.05)$ cells (Figure $2(\mathrm{a}))$. To detect the significant roles of miR-193a, miR-193a mimic and miR-193a inhibitor were conducted to upregulate $(P<0.01)$ or downregulate $(P<0.05)$ miR-193a in HGC-27 cells measured by RT-qPCR (Figure 2(b)).

CCK8 assay indicated that miR-193a mimic suppressed $(P<0.05)$ cell proliferation, while miR-193a inhibitor 


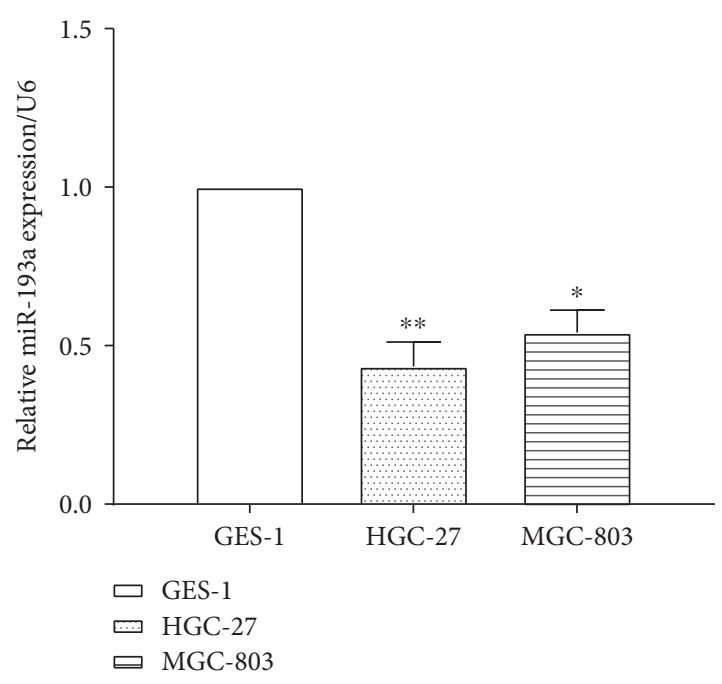

(a)

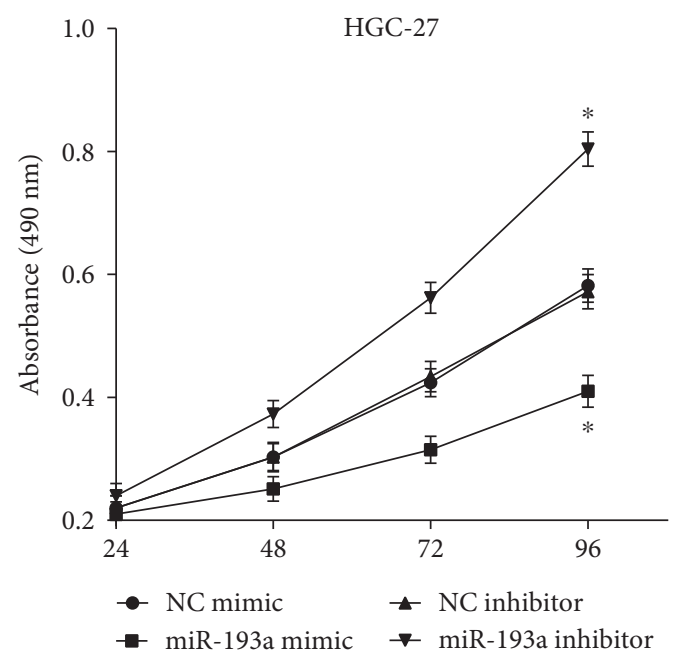

(c)

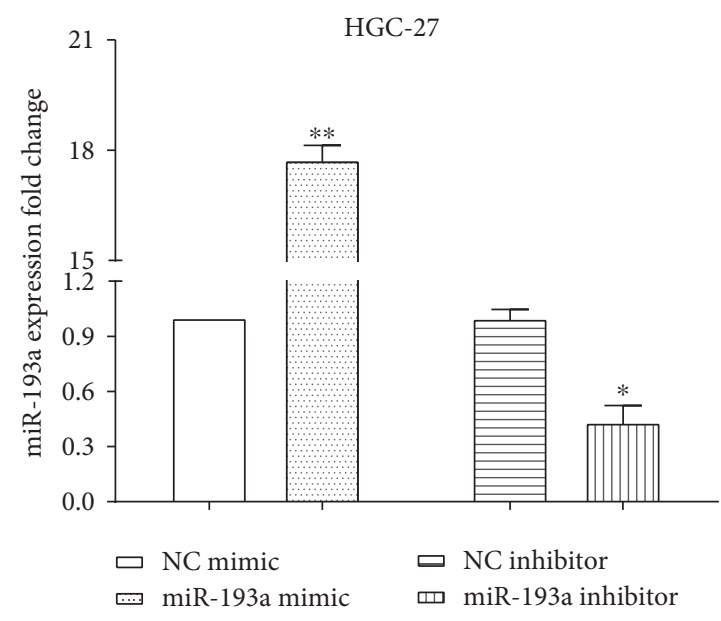

(b)

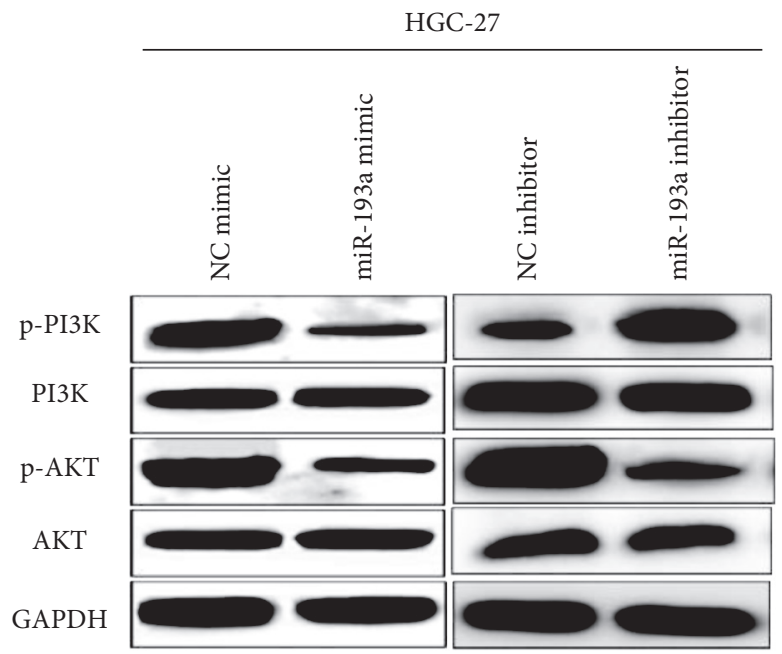

(d)

FIGURE 2: miR-193a suppresses the activation of PI3K/AKT pathway in gastric cancer cells. (a) miR-193a expression was low in GES-1 cells versus HGC-27 and MGC-803 cells. (b) The miR-193a mimic and miR-193a inhibitor were conducted to upregulate or downregulate miR193a in HGC-27 cells measured by RT-qPCR. (c) CCK8 assay indicated miR-193a mimic suppressed cell proliferation, while miR-193a inhibitor promoted cell proliferative ability in HGC-27 cells. (d) miR-193a inhibited the activation of PI3K/AKT pathway. ${ }^{*} P<0.05$; ${ }^{* *} P<0.01$.

promoted $(P<0.05)$ cell proliferative ability in HGC-27 cells (Figure 2(c)). Western blot assay was conducted to assess the expression of PI3K pathway-associated proteins in HGC-27 cells. We discovered that miR-193a overexpression suppressed p-PI3K and p-AKT expression in HGC-27 cells, whereas miR-193a inhibitor enhanced the expression of p-PI3K and p-AKT, which elucidated that miR-193a inhibited the activation of PI3K/Akt signaling pathway (Figure 2(d)).

3.3. miR-193a Suppresses the Gastric Cancer Growth In Vivo. HGC-27 cells stably transfected with miR-193a mimic or control plasmid were applied to inject into the nude mice at subcutaneous. The xenograft tumors volumes were calculated every 3 days and the group of transfecting miR-193a mimic had a slower growth rate than the control group (Figure 3(a)). After 26 days of culture, the nude mice were dissected and the tumor volume was calculated and recorded. Furthermore, we discovered that the tumor volumes in miR-193a overexpression group were smaller than those in control group, which indicated that overexpressed miR-193a inhibited gastric cancer growth in vivo $(P<0.05)$ (Figure 3(b)).

3.4. miR-193a Inhibits Cell Invasion and the EMT in HGC-27 Cells. Transwell assay elucidated that miR-193a mimic suppressed $(P<0.05)$ cell invasive ability whereas miR-193a inhibitor enhanced cell invasive ability $(P<0.05)$ (Figure 4(a)). All the findings revealed miR-193a inhibited the abilities of proliferation and invasion in gastric cancer 


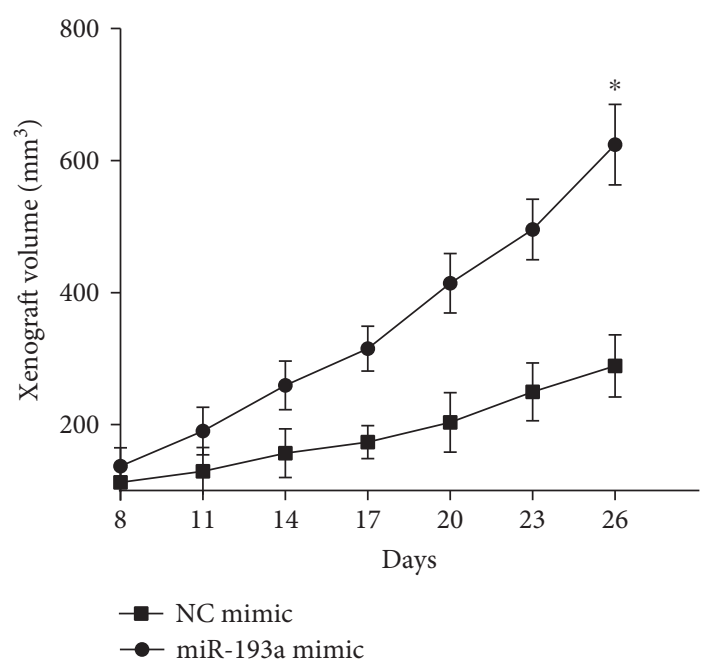

(a)

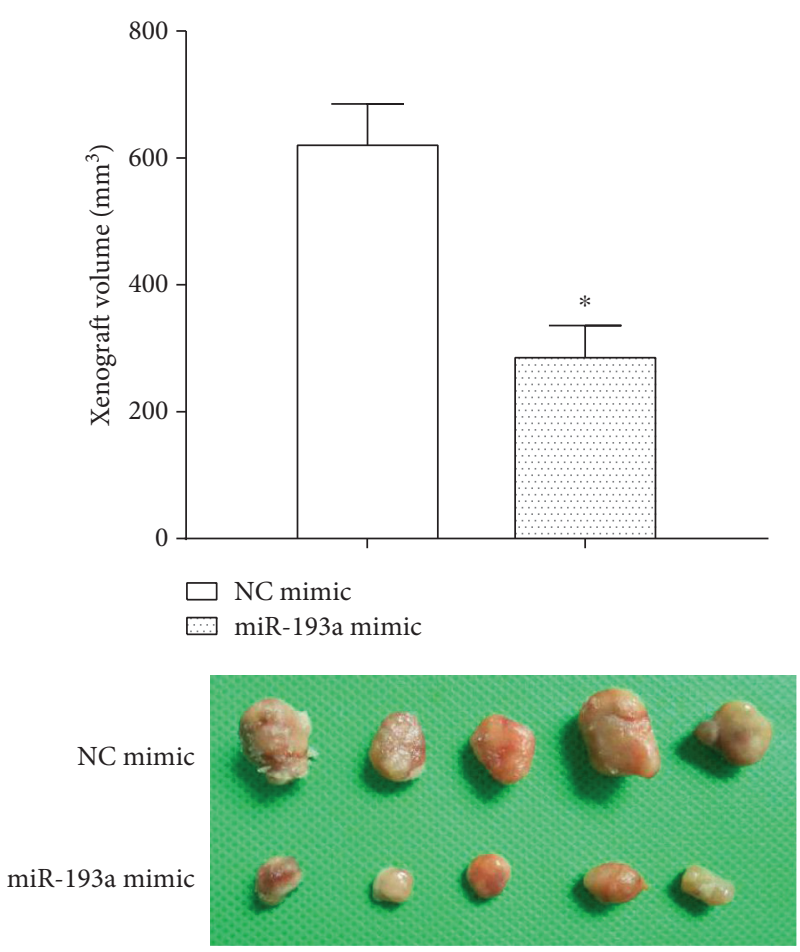

(b)

FIGURE 3: miR-193a suppresses the xenograft growth in vivo. (a) miR-193a overexpression inhibited gastric cancer xenograft growth. (b) The tumor volume of cells overexpressed miR-193a was smaller than the control group. ${ }^{*} P<0.05$.
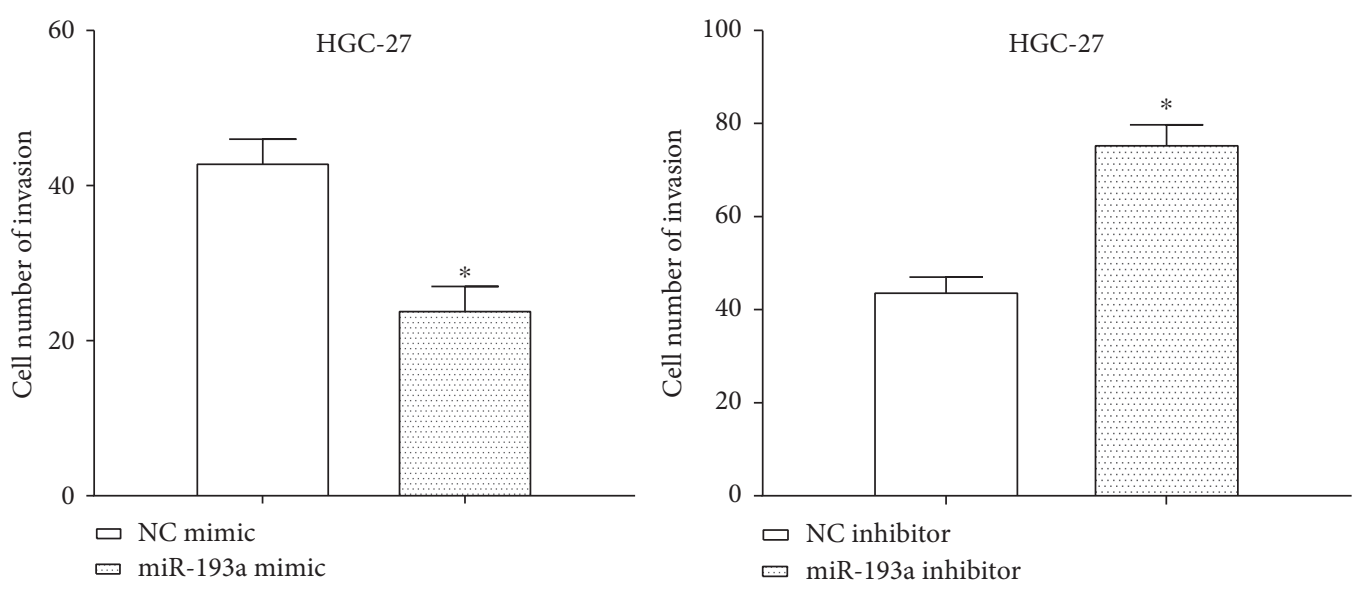

HGC-27

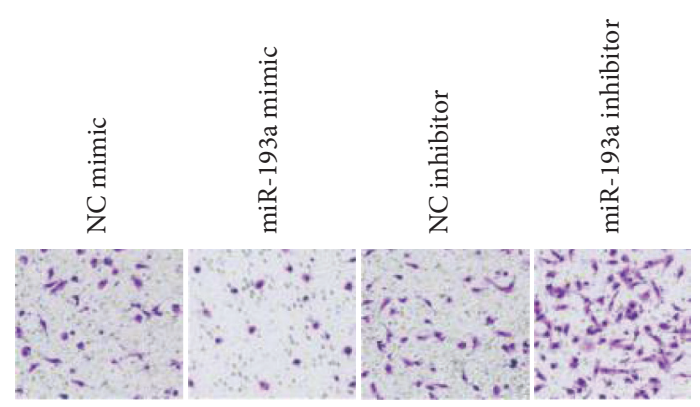

(a)

Figure 4: Continued. 


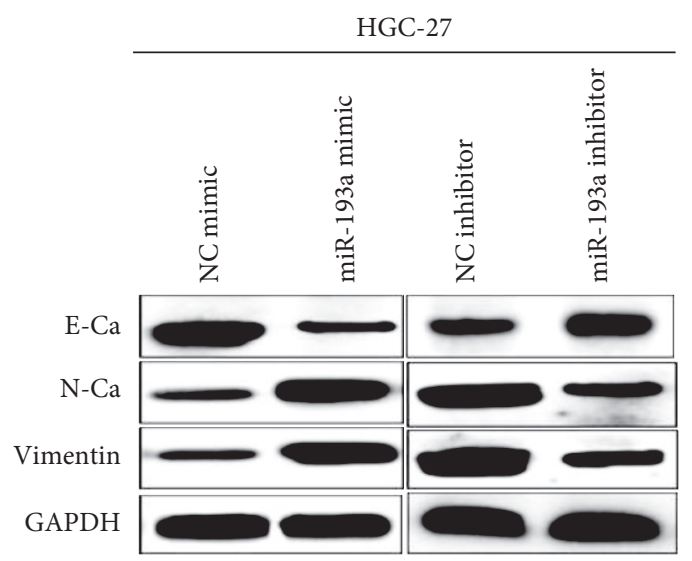

(b)

FIGURE 4: miR-193a suppresses cell invasion and EMT in gastric cancer cells. (a) Transwell assay elucidated that miR-193a inhibited the abilities of invasion in gastric cancer cell HGC-27. (b) miR-193a inhibited the EMT in HGC-27 cells. ${ }^{*} P<0.05$.

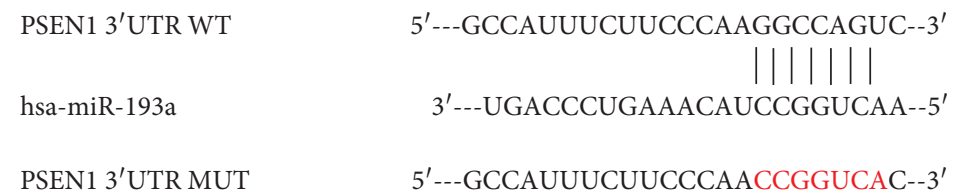

(a)

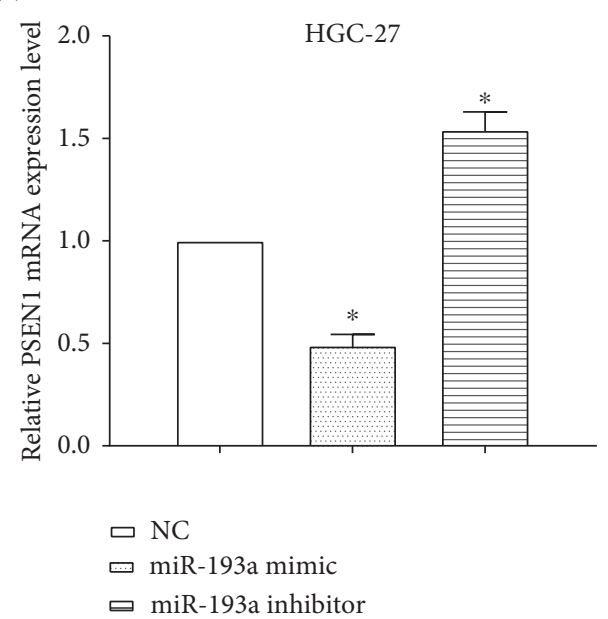

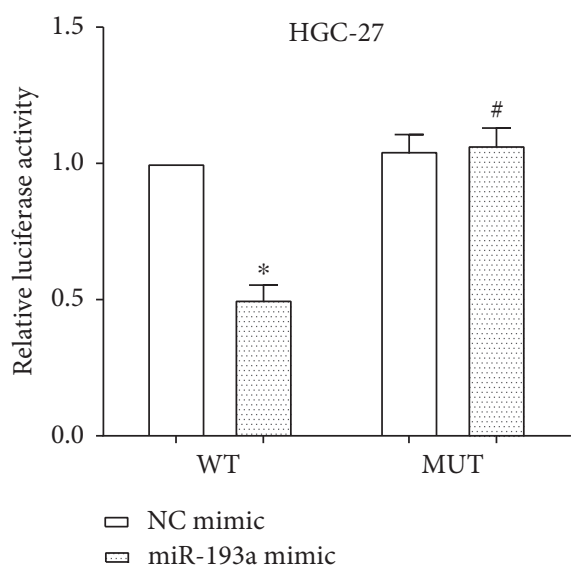

(b)

(c)

FIGURE 5: miR-193a regulates PSEN1 expression through binding to PSEN1 mRNA 3'-UTR. (a) PSEN1 was a target gene of miR-193a, and the binding site was located at the $3^{\prime}$-UTR of PSEN1 mRNA. (b) Luciferase reporter assay elucidated miR-193a and decreased the luciferase activity of HGC-27 cells transfected with wild-type PSEN1 $3^{\prime}$-UTR, while it did not alter the luciferase activity of cells transfected with mutant PSEN1 3'-UTR. (c) Overexpression of miR-193a inhibited the mRNA level of PSEN1, while knockdown of miR-193a enhanced the expression of PSEN1 in HGC-27 cells. ${ }^{*} P<0.05 ;{ }^{\#} P<0.05$. 


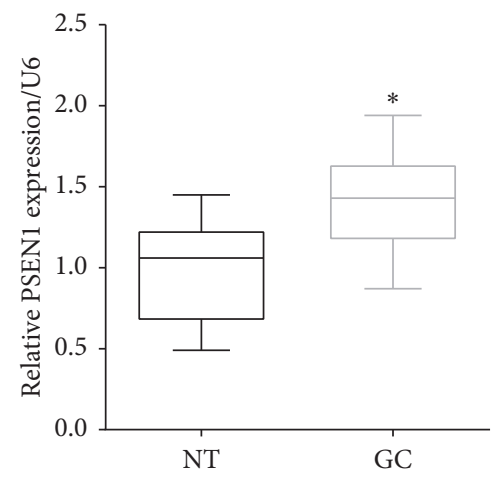

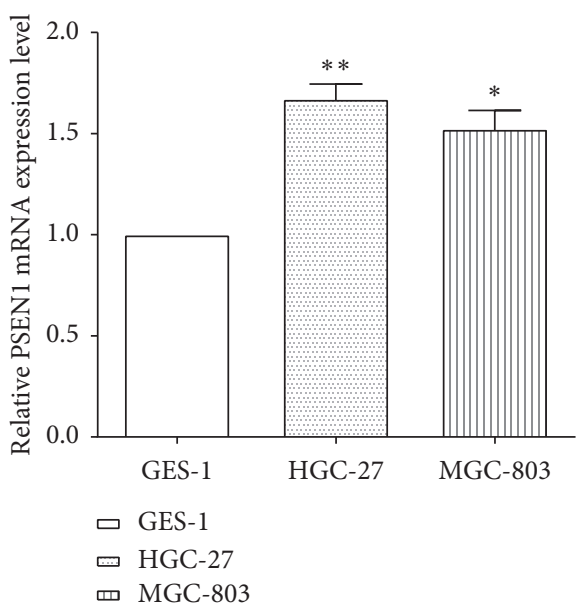

(b)

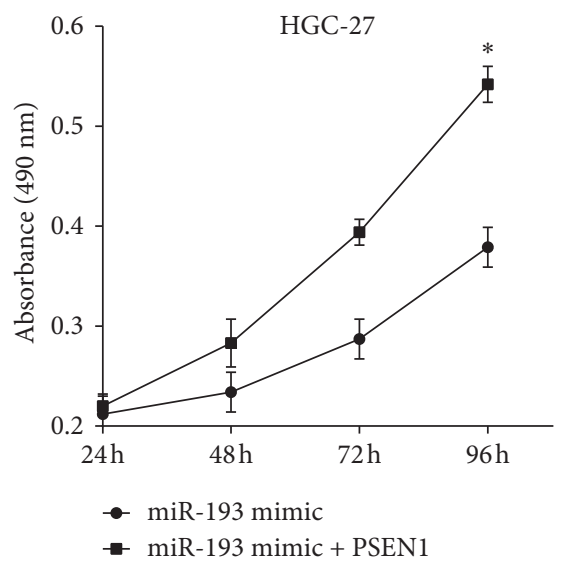

HGC-27

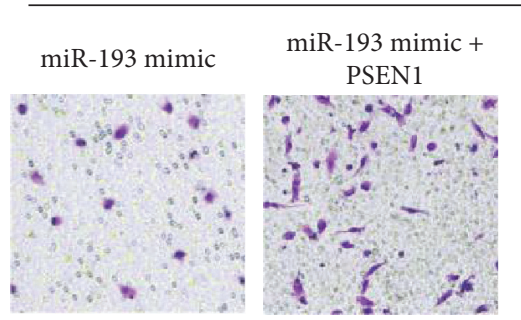

(d)

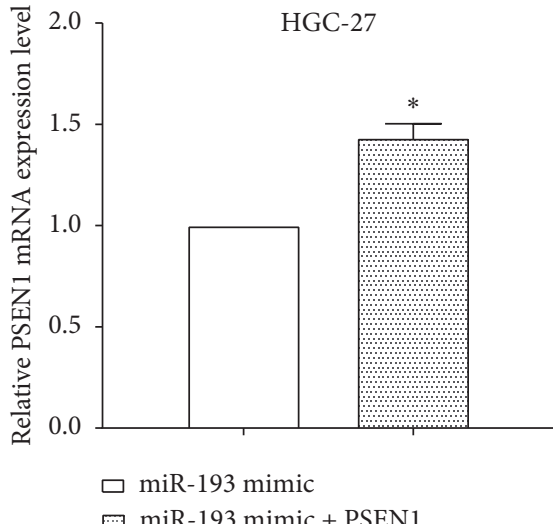

$\square$ miR-193 mimic + PSEN1

Figure 6: Continued. 


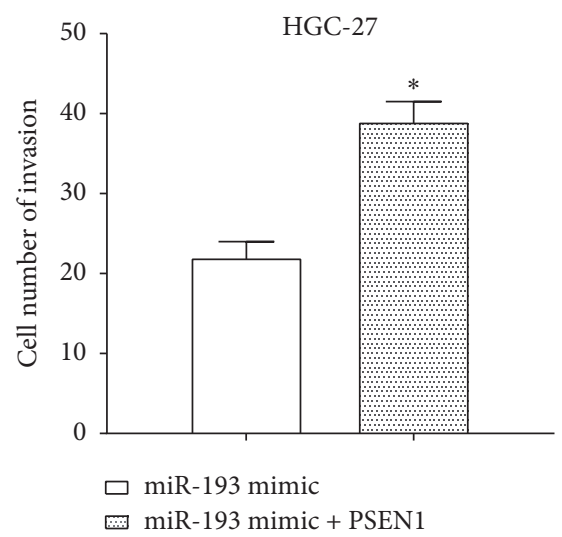

(e)

FIGURE 6: PSEN1 reverses the roles of miR-193a on cell proliferation and invasion. (a) PSEN1 was upregulated in gastric cancer tissues compared to peritumoral normal tissues. (b) PSEN1 expression was higher in HGC-27 and MGC-803 cells than that in GES-1 cells. (c) PSEN1 overexpression plasmid was transfected in miR-193a overexpressed HGC-27 cells. (d) Upregulation of PSEN1 enhanced cell proliferation in miR-193a mimictransfected HGC-27 cells. (e) PSEN1 reversed partial roles of miR-193a on cell invasion. ${ }^{*} P<0.05 ;{ }^{* *} P<0.01$.

cell HGC-27. The EMT ability was evaluated by detecting the expression of EMT-associated proteins by western blot. As we expected, miR-193a mimic suppressed the expression of $\mathrm{N}$-cadherin, while improving the expression of E-cadherin in HGC-27 cells. On the contrary, miR-193a inhibitor enhanced the expression of $\mathrm{N}$-cadherin and decreased E-cadherin expression, which suggested that miR-193a inhibited cell EMT through PSEN1 (Figure 4(b)).

3.5. miR-193a Regulates the Expression of PSEN1 through Directly Binding to 3'-UTR of PSEN1 mRNA. TargetScan predicted one of the target genes of miR-193a was PSEN1, and the binding site was located at $697-703$ on PSEN1 mRNA 3'-UTR. To verify miR-193a binding to the potential binding site of PSEN1, the binding sequences were mutated from GGCCAGU to CCGGUCA, and then the luciferase activity was calculated (Figure 5(a)). The luciferase reporter assay elucidated that miR-193a decreased $(P<0.05)$ the luciferase activity of HGC-27 cells transfected with wild-type PSEN1 3 '-UTR, while it did not alter $(P>0.05)$ the luciferase activity of cells transfected with mutant PSEN1 $3^{\prime}$-UTR (Figure 5(b)). PSEN1 mRNA levels were evaluated after transfection of miR-193a mimic or miR-193a inhibitor in HGC-27 cells. As expected, overexpression of miR-193a inhibited $(P<0.05)$ the mRNA level of PSEN1, while knockdown of miR-193a enhanced $(P<0.05)$ PSEN1 expression in HGC-27 cells (Figure 5(c)). All the results indicated that miR-193a regulated the expression of PSEN1 in gastric cancer cells HGC-27.

3.6. PSEN1 Reverses the Roles of miR-193a in Gastric Cancer Development. The expression of PSEN1 was evaluated by RT-qPCR and we found that it was downregulated in peritumoral normal tissues compared to that in gastric cancer tissues $(P<0.05)$ (Figure 6(a)). Similarly, the expression of PSEN1 was higher in HGC-27 $(P<0.01)$ and
MGC-803 $(P<0.05)$ cells than that in GES-1 cells (Figure 6(b)).

To investigate the effect of PSEN1 on the suppressive role of miR-193a, PSEN1 overexpression plasmid was transfected in miR-193a overexpressed HGC-27 cells (Figure 6(c)). Upregulation of PSEN1 enhanced cell proliferation and invasion in miR-193a mimic-transfected HGC-27 cells (Figures 6(d) and 6(e)). All the results revealed that PSEN1 partially reversed the roles of miR-193a on cell proliferation and invasion.

\section{Discussion}

Gastric cancer, a kind of gastrointestinal malignancy, is the second leading death cancer, and the early diagnosis is very difficult $[1,2]$. Thus, exploring new biomarkers for the early diagnosis and treatment of gastric cancer is urgent.

Micro-RNAs caused mRNA degradation or translational inhibition through binding to target mRNAs $3^{\prime}$ UTR at the posttranscriptional level [21, 22]. Accumulating evidence indicated that multiple miRNAs were involved in tumor prognosis in gastric cancer, including miR-127, miR-575, miR-31, and miR-520c [23-26]. miR193a acted as a key tumor suppressor in various cancers and played significant roles in tumor growth and metastasis $[8,11]$. miR-193a inhibited cell viability, proliferation, and colony formation and induced G1 phase arrest in prostate cancer cells [27]. Consistent with all the findings above, we discovered that miR-193a was downregulated in gastric cancer tissues and cells, and downregulation of miR-193a predicted poor 5-year overall survival of gastric cancer patients. miR-193a inhibited tumor growth in vitro and in vivo and suppressed tumor metastasis in gastric cancer cells HGC-27. Pan et al. indicated that miR-193a inhibited cell proliferation and invasion through PI3K/AKT pathway in renal cell carcinoma [28]. Our results are consistent with Pan 
et al.; miR-193a suppressed the activation of PI3K/AKT pathway in gastric cancer. What is more, we first propose that miR-193a inhibited the epithelial-to-mesenchymal transition (EMT) of gastric cancer cells. However, miR193a promoted cell proliferation and migration in esophageal squamous cell carcinoma [29]; therefore, we conjectured that miR-193a might have tissue specificity.

PSEN1 is a primary component of the $\gamma$-secretase complex, which is mainly related to Alzheimer's disease $[14,15]$. PSEN1 downregulation caused decreased neuronal survival and protected neurons from glucose deprivation-induced death [30]. Even in gastric cancer, Li et al. indicated that PSEN1 enhanced carcinogenesis and metastasis [31]. PSEN1 is a direct target gene of miR193a, and PSEN1 promoted cell apoptosis and enhanced the sensitization to a drug in bladder cancer [32]. Consistent with Meng et al. $[18,32]$, we found that PSEN1 was a target of miR-193a and its expression was mediated by miR-193a in gastric cancer cells HGC-27. PSEN1 partially reversed the functions of miR-193a in cell proliferation and invasion in HGC-27 cells. The shortcoming of our study is that it has not further explored the mechanism of the miRNA/PSEN1/PI3K/Akt axis in gastric cancer.

\section{Conclusions}

miR-193a inhibits gastric cancer cell proliferation, invasion, and the epithelial-to-mesenchymal transition and suppresses the activation of the PI3K/Akt signaling pathway. This study evaluates the therapeutic potential of miR-193a in gastric cancer and provides a new target for the prevention and treatment of gastric cancer.

\section{Data Availability}

The datasets used and/or analyzed during the present study are available from the corresponding author on reasonable request.

\section{Ethical Approval}

The study was approved by the Qingdao Hospital of Traditional Chinese Medicine.

\section{Consent}

Written permission was obtained from all participants.

\section{Disclosure}

The funding body had no role in the design of the study, collection, analysis, interpretation of data, or writing of the manuscript.

\section{Conflicts of Interest}

The authors declare that they have no conflicts of interest.

\section{Authors' Contributions}

$\mathrm{XP}$ contributed to the conception, design, experiments, and drafting of the article. TZ and SM contributed to the collection of human samples, data analysis, and statistical analysis. SL contributed to the conception, interpretation of data, and critical revision of the manuscript.

\section{Acknowledgments}

The authors deeply appreciate the contributions to this work made in various ways by all of the participants.

\section{References}

[1] A. Jemal, F. Bray, M. M. Center, J. Ferlay, E. Ward, and D. Forman, "Global cancer statistics," CA: A Cancer Journal for Clinicians, vol. 61, no. 2, pp. 69-90, 2011.

[2] W.-M. Chen, M.-D. Huang, D.-P. Sun et al., "Long intergenic non-coding RNA 00152 promotes tumor cell cycle progression by binding to EZH 2 and repressing $\mathrm{p} 15$ and $\mathrm{p} 21$ in gastric cancer," Oncotarget, vol. 7, no. 9, pp. 9773-9787, 2016.

[3] Y. Yang, Y. Liu, W. Liu et al., "miR-122 inhibits the cervical cancer development by targeting the oncogene RAD21," Biochemical Genetics, 2021, Internet.

[4] X. Lin, Z. Yang, P. Zhang, Y. Liu, and G. Shao, "[Retracted] miR-154 inhibits migration and invasion of human non-small cell lung cancer by targeting ZEB2," Oncology Letters, vol. 22, no. 2, p. 612, 2021.

[5] Y. Saito, H. Suzuki, and T. Hibi, "The role of microRNAs in gastrointestinal cancers," Journal of Gastroenterology, vol. 44, no. Suppl 19, pp. 18-22, 2009.

[6] T. Ueda, S. Volinia, H. Okumura et al., "Relation between microRNA expression and progression and prognosis of gastric cancer: a microRNA expression analysis," The Lancet Oncology, vol. 11, no. 2, pp. 136-146, 2010.

[7] F. Xiao, Z Zuo, G Cai, S Kang, X Gao, and T Li, "miRecords: an integrated resource for microRNA-target interactions," Nucleic Acids Research, vol. 37, no. Database issue, pp. D105-D110, 2009.

[8] J. Pekow, K. Meckel, U. Dougherty et al., "miR-193a-3p is a key tumor suppressor in ulcerative colitis-associated colon cancer and promotes carcinogenesis through upregulation of IL17RD," Clinical Cancer Research, vol. 23, no. 17, pp. 5281-5291, 2017.

[9] X.-N. Gao, J. Lin, Y.-H. Li et al., "MicroRNA-193a represses c-kit expression and functions as a methylation-silenced tumor suppressor in acute myeloid leukemia," Oncogene, vol. 30, no. 31, pp. 3416-3428, 2011.

[10] Y. Li, H. Deng, L. Lv et al., "The miR-193a-3p-regulated ING5 gene activates the DNA damage response pathway and inhibits multi-chemoresistance in bladder cancer," Oncotarget, vol. 6, no. 12, pp. 10195-10206, 2015.

[11] M. Williams, M. B. Kirschner, Y. Y. Cheng et al., "miR-193a$3 \mathrm{p}$ is a potential tumor suppressor in malignant pleural mesothelioma," Oncotarget, vol. 6, no. 27, pp. 23480-23495, 2015.

[12] T. Yu, J. Li, M. Yan et al., "MicroRNA-193a-3p and $-5 p$ suppress the metastasis of human non-small-cell lung cancer by downregulating the ERBB4/PIK3R3/mTOR/S6K2 signaling pathway," Oncogene, vol. 34, no. 4, pp. 413-423, 2015.

[13] C. Fang, C.-Y. Dai, Z. Mei et al., "microRNA-193a stimulates pancreatic cancer cell repopulation and metastasis through 
modulating TGF- $\beta 2 /$ TGF- $\beta$ RIII signalings," Journal of Experimental and Clinical Cancer Research, vol. 37, no. 1, p. 25, 2018.

[14] H. Jin, N. Sanjo, T. Uchihara et al., "Presenilin-1 holoprotein is an interacting partner of sarco endoplasmic reticulum calcium-ATPase and confers resistance to endoplasmic reticulum stress," Journal of Alzheimer's Disease, vol. 20, no. 1, pp. 261-273, 2010.

[15] J. Wang, R. Lu, J. Yang et al., “TRPC6 specifically interacts with APP to inhibit its cleavage by $\gamma$-secretase and reduce $\mathrm{A} \beta$ production," Nature Communications, vol. 6, no. 1, p. 8876, 2015.

[16] Y. Takahashi, Y. Ohta, R. Sasaki et al., "A novel presenilin 1 mutation (Leu418Trp) associated with spasticity, parkinsonism, and white matter lesion in a dominant alzheimer's family," Journal of the Neurological Sciences, vol. 387, pp. 166-169, 2018.

[17] Y. Zhao, X. Li, T. Huang et al., "Intracellular trafficking of TREM2 is regulated by presenilin 1," Experimental and Molecular Medicine, vol. 49, no. 12, p. e405, 2017.

[18] F. Meng, L. Qian, L. Lv et al., "miR-193a-3p regulation of chemoradiation resistance in oesophageal cancer cells via the PSEN1 gene," Gene, vol. 579, no. 2, pp. 139-145, 2016.

[19] S. Wang, Y. Wu, S. Yang et al., "miR -874 directly targets AQP3 to inhibit cell proliferation, mobility and EMT in nonsmall cell lung cancer," Thoracic Cancer, vol. 11, no. 6, pp. 1550-1558, 2020.

[20] D. D. Zhou, H. L Li, W. Liu et al., "miR-193a-3p promotes the invasion, migration, and mesenchymal transition in glioma through regulating BTRC," BioMed Research International, vol. 2021, Article ID 8928509, 22 pages, 2021.

[21] E. C. Lai, "Micro RNAs are complementary to 3 ' UTR sequence motifs that mediate negative post-transcriptional regulation," Nature Genetics, vol. 30, no. 4, pp. 363-364, 2002.

[22] A. Gargalionis and E. Basdra, "Insights in microRNAs biology," Current Topics in Medicinal Chemistry, vol. 13, no. 13, pp. 1493-1502, 2013.

[23] L. Wang, X. Wang, and X. Jiang, "miR-127 suppresses gastric cancer cell migration and invasion via targeting Wnt7a," Oncology Letters, vol. 17, no. 3, pp. 3219-3226, 2019.

[24] Y.-N. Wang, F. Xu, P. Zhang et al., "MicroRNA-575 regulates development of gastric cancer by targeting PTEN," Biomedicine and Pharmacotherapy, vol. 113, Article ID 108716, 2019.

[25] A. Korourian, Z. Madjd, R. Roudi, A. Shariftabrizi, and M. Soleimani, "Induction of miR-31 causes increased sensitivity to 5-FU and decreased migration and cell invasion in gastric adenocarcinoma," Bratislavské lekárske listy, vol. 120, no. 1, pp. 35-39, 2019.

[26] A. Azimzadeh-Isfanjani, R. Safaralizadeh, M. HosseinpourFeizi, B. Shokouhi, M. Nemati, and S.-Y. Moaddab, "Expression of miR-520c in intestinal type gastric adenocarcinoma," Journal of Gastrointestinal Oncology, vol. 9, no. 6, pp. 1184-1189, 2018.

[27] Y. Liu, X Xu, X. Xu et al., "MicroRNA-193a-3p inhibits cell proliferation in prostate cancer by targeting cyclin D1," Oncology Letters, vol. 14, no. 5, pp. 5121-5128, 2017.

[28] Y. Pan, J. Hu, J. Ma et al., "MiR-193a-3p and miR-224 mediate renal cell carcinoma progression by targeting alpha-2,3-sialyltransferase IV and the phosphatidylinositol 3 kinase/Akt pathway," Molecular Carcinogenesis, vol. 57, no. 8, pp. 1067-1077, 2018.

[29] Y. Yi, J. Chen, C. Jiao et al., "Upregulated miR-193a-3p as an oncogene in esophageal squamous cell carcinoma regulating cellular proliferation, migration and apoptosis," Oncology Letters, vol. 12, no. 6, pp. 4779-4784, 2016.

[30] Q. Huang, G. Voloudakis, Y. Ren et al., "Presenilin $1 / \gamma$-secretase protects neurons from glucose deprivation-induced death by regulating miR-212 and PEA15," The FASEB Journal, vol. 32, no. 1, pp. 243-253, 2018.

[31] P. Li, X. Lin, J.-R. Zhang et al., "The expression of presenilin 1 enhances carcinogenesis and metastasis in gastric cancer," Oncotarget, vol. 7, no. 9, pp. 10650-10662, 2016.

[32] H. Deng, L. Lv, Y. Li et al., "The miR-193a-3p regulated PSEN1 gene suppresses the multi-chemoresistance of bladder cancer," Biochimica et Biophysica Acta (BBA) - Molecular Basis of Disease, vol. 1852, no. 3, pp. 520-528, 2015. 